\title{
Impact of therapy dog visits on outpatient nurse welfare and job satisfaction
}

\author{
Stephanie D. Clark*, Jessica M. Smidt, and Brent A. Bauer
}

\section{Abstract}

Interaction with a therapy dog can decrease blood pressure, heart rate, and improve heart rate variability; due to these responses, it suggests that human-animal interaction can alleviate the stress response. This study aims to observe if the effects of therapy dog visits could alleviate nursing burnout and increase work satisfaction in an outpatient setting. In addition, this study will observe at what visit frequency of therapy dog visits nurses benefited from most. This study is a two-part study, which also observed the salivary cortisol concentrations of the therapy dogs post therapy visit interaction. The study design was a controlled before-after study design with five treatments over the course of six months, each treatment (TRT) lasting four weeks: TRT A, two therapy dog visits a week; TRT B, one visit a week; TRT C, two visits; TRT D, one visit; and TRT E, no visits. Four out-patient nursing units were selected and asked to complete a demographic survey, the Pet Attitude Scale-Modified, and Lexington Attachment to Pet Scale. Pre- and post-treatments, participants completed the Human Services Survey, Nursing Workplace Satisfaction Questionnaire, Nursing Work Index (Revised), and a visual analog scale. TRT A was able to significantly increase the feeling of happiness. In addition TRT $\mathrm{B}$, a therapy dog visit once a week, was able to significantly reduce self-reported responses of depression and improve emotional wellbeing. Consequently, TRT E, control/no therapy dog visits, had the least amount of improvement in the nursing units' visual analog scale. This study supports the hypothesis that therapy dog visits can help alleviate stress, frustration, feeling drained, and the overwhelming sensation that can come from working in the nursing field.

Pet Behaviour Science | 2018, Vol.6, 8 - 15 DOI: $10.21071 /$ pbs.v0i6.11172

\section{Stephanie D. Clark*, Jessica M. Smidt, and} Brent A. Bauer

* Department of Integrative Medicine and Health Division of General Internal

Medicine

Mayo Clinic

200 First St SW

Rochester, MN 55905
Research paper

* Email: clark.stephanie1@mayo.edu

Keywords:

Burnout; compassion fatigue; therapy dogs; welfare

\section{Highlights}

- Outpatient nursing units that experience a therapy dog visit, once a week, self-reported an improvement in emotional wellbeing score, including, depression, stress, happiness, energy level, relaxation, calmness, and overall emotional wellbeing..

- No therapy dog visit group, had the least amount of improvement in the nursing units' visual analog scale was seen, with six parameters, restlessness, anxiety, depression, relaxation, calmness, and overall emotional wellbeing, being self-reported as worse. 
- Participated in the study who currently owned a pet $(100 \%)$, over $80 \%$ believe that owning pets contribute to their happiness.

\section{INTRODUCTION}

Nurse and health care provider burnout has consistently been an upward battle. Burnout is described as a syndrome characterized by emotional exhaustion, depersonalization, and a decrease in personal accomplishment (Vahey et al., 2004). In addition to decreases in productivity and patient satisfaction, burnout can also lead to psychological distress, an increase in reported complaints, and drug and alcohol abuse (Vahey et al., 2004). Reports state that over $40 \%$ of nurses experience burnout, a higher rate than other health care providers is often a result of unrealistic workloads Aiken et al., 2011). While nurses play a primary role in the overall care of patients and daily hospital tasks, the nursing field is inadequately staffed and a main limiting factor in high-quality hospital care (Aiken et al., 2002). In addition, these nurses indicated that their stress-coping behaviors included exercise, social support, verbal venting, selfdistraction, and self-blame (Shimizutani et al., 2008). In addition to self-care behavior, another strategy that can be easily implemented to decrease nurse burnout is animal-assisted therapy visits.

The human-animal bond is growing stronger and the demand for therapy dog visits in a hospital setting is increasing. Studies have been conducted observing the beneficial relationship animals have on the elderly, children with autism, hospitalized patients, hospice care, and behavioral conditions (Nimer and Lundahl, 2007). In particular, one study observed the therapeutic effects of dog visits with hospitalized patients with a psychiatric disorder. The researchers observed that a dog visit significantly reduced the level of anxiety experienced by patients (Barker and Dawson, 1998). Additionally, animal assisted therapy visits can improve quality of life, coping ability, and self-efficacy (Berget et al., 2008). Animal assisted therapy not only improves mental outlook, but physiologically, 20 minutes of petting a dog can increase oxytocin concentrations, as well as positively affect other endocrine responses, such as a decrease in cortisol, epinephrine, and norepinephrine (Beetz et al., 2012).
Interaction with a friendly animal can also decrease blood pressure, heart rate, and improve heart rate variability; due to these responses, it suggests that human animal interaction can alleviate a stress response (Beetz et al., 2012). With this supporting data, animal-assisted therapy visits could provide an easily accessible and minimal time-invested therapy that could potentially alleviate nursing burnout and increase quality of care.

\section{METHODS}

\section{Participants}

Four outpatient nursing units in the General Internal Medicine Department at Mayo Clinic, Rochester, MN were selected for participation in this study. The four nursing units were selected based upon the nurse population that worked full time, did not work in other units, and were able to participate in all five treatments. This selection criterion for the nursing units was applied to ensure that nurses would not see other therapy dogs at different frequencies, but that the nurses would be available during the visits. The nursing units within the Department of General Internal Medicine included 1) women's health and consulting medicine; 2) home enteral nutrition and home paracentral nutrition; and 3 and 4) two different departments of executive health. Each nursing unit had six nurses participating in the study. A total of 24 nurses participated, 23 women and 1 man, with an average age of $43.13 \pm S D=11.76$ years with $20.29 \pm S D$ $=11.78$ years of experience (Table 1). All nurses participating in the study signed a consent form and a Health Insurance Portability and Accountability Act (HIPAA) waiver before completing any questionnaires or visiting with any therapy dogs. 


\begin{tabular}{|c|c|c|c|c|c|c|c|c|c|}
\hline Nurse & Age & Sex & $\begin{array}{l}\text { Marital } \\
\text { Status }\end{array}$ & Race & Smoker & $\begin{array}{l}\text { Own } \\
\text { a Pet }\end{array}$ & $\begin{array}{c}\text { Nursing } \\
\text { Unit }\end{array}$ & $\begin{array}{l}\text { Years of } \\
\text { Nursing }\end{array}$ & $\begin{array}{c}\text { Years in } \\
\text { Present } \\
\text { Unit }\end{array}$ \\
\hline \multicolumn{10}{|l|}{$\operatorname{Dog} 1^{*}$} \\
\hline 1 & 41 & F & Married & White & $\mathrm{N}$ & Y & HEN & 21 & 3 \\
\hline 2 & 39 & F & Single & White & $\mathrm{N}$ & Y & HEN & 17 & 0.5 \\
\hline 3 & 40 & F & Divorced & White & $\mathrm{N}$ & $\mathrm{Y}$ & HPN & 19 & 3 \\
\hline 4 & 45 & F & Divorced & White & $\mathrm{N}$ & $\mathrm{Y}$ & HPN & 22 & 2 \\
\hline 5 & 34 & F & Married & White & $\mathrm{N}$ & $\mathrm{Y}$ & HPN & 11 & 0.5 \\
\hline 6 & - & F & - & White & $\mathrm{N}$ & $\mathrm{Y}$ & HPN & - & - \\
\hline \multicolumn{10}{|l|}{$\operatorname{Dog} 2$} \\
\hline 7 & 23 & $\mathrm{~F}$ & Single & White & $\mathrm{N}$ & Y & WHC & 1 & 1 \\
\hline 8 & 54 & F & Married & White & $\mathrm{N}$ & $\mathrm{N}$ & WHC & 30 & 9 \\
\hline 9 & 30 & F & Married & White & $\mathrm{N}$ & $\mathrm{Y}$ & WHC & 7.5 & 1.3 \\
\hline 10 & 63 & F & Married & White & $\mathrm{N}$ & $\mathrm{Y}$ & $\mathrm{BDC}$ & 45 & 1.5 \\
\hline 11 & 40 & F & Married & White & $\mathrm{N}$ & $\mathrm{Y}$ & BDC & 18 & 3 \\
\hline 12 & 47 & F & - & White & $\mathrm{N}$ & $\mathrm{Y}$ & $\mathrm{BDC}$ & 24 & 7 \\
\hline \multicolumn{10}{|l|}{$\operatorname{Dog} 3$} \\
\hline 13 & 43 & F & Married & White & $\mathrm{N}$ & Y & EXEC & 21 & 5 \\
\hline 14 & 55 & F & Married & White & $\mathrm{N}$ & $\mathrm{Y}$ & EXEC & 30 & 2 \\
\hline 15 & 58 & F & Married & White & $\mathrm{N}$ & $\mathrm{Y}$ & EXEC & 38 & 1 \\
\hline 16 & 46 & F & Married & White & $\mathrm{N}$ & $\mathrm{N}$ & EXEC & 23 & 1 \\
\hline 17 & 56 & F & Married & White & $\mathrm{N}$ & $\mathrm{Y}$ & EXEC & 34 & 1 \\
\hline 18 & 34 & F & Married & White & $\mathrm{Y}$ & $\mathrm{Y}$ & EXEC & 13 & 2 \\
\hline \multicolumn{10}{|l|}{$\operatorname{Dog} 4$} \\
\hline 19 & 32 & $\mathrm{M}$ & Single & White & $\mathrm{N}$ & $\mathrm{Y}$ & EXEC & 5 & 2 \\
\hline 20 & 58 & $\mathrm{~F}$ & Married & White & $\mathrm{Y}$ & $\mathrm{Y}$ & EXEC & 23 & 4 \\
\hline 21 & 28 & F & Married & White & $\mathrm{N}$ & $\mathrm{Y}$ & EXEC & 6 & 2 \\
\hline 22 & 26 & F & Single & White & $\mathrm{N}$ & $\mathrm{Y}$ & EXEC & 4 & 2.5 \\
\hline 23 & 56 & F & Married & White & - & $\mathrm{Y}$ & EXEC & 34 & 0.5 \\
\hline 24 & 55 & $\mathrm{~F}$ & Married & White & $\mathrm{N}$ & $\mathrm{Y}$ & EXEC & 30 & 2.5 \\
\hline
\end{tabular}

Table 1. Sociodemographics of the Out-Patient Nurses. BDC, Breast Diagnostic Clinic; EXEC, Executive Health; HEN, Home Enteral Nutrition Program; HPN, Home Parenteral Nutrition Program; WHC, Women's Health Clinic. "Therapy dog that visited out-patient nurses for the duration of the project.

Therapy Dogs

Four therapy dog teams from Mayo Clinic, Rochester's Caring Canine Program volunteered for this study. Each therapy dog team is an active volunteer, seeing patients at the clinic regularly, registered with a therapy dog organization, and were willing to stop additional therapy dog visits for the duration of this study. The demographics for the four dogs were two males, a 12 yr old Bichon (Dog 1) and a 10 yr old small mixed breed (Dog 2); and two females, a 7 yr old
Standard Poodle (Dog 3) and a 10 yr old Shi-poo (Dog 4). Both male dogs had female handlers and were registered with Pet Partners. In contrast, both female dogs had male handlers; however, Dog 3 was registered with Therapy Dog International and Dog 4 was registered with Alliance of Therapy Dogs. Mayo Clinic Rochester's IACUC committee approved this study (protocol A00003246-17). 


\section{Study Design}

The study was designed as a controlled before-after study for the nursing units experiencing therapy dog visits. The four therapy dog teams were randomly assigned to a nursing unit, by being asked to pick an envelope that contained the nursing unit they would be visiting. The therapy dog teams were then randomly assigned to the order of the four treatments using PROC PLAN block randomization design on SAS version 9.4. The treatments included treatment (TRT) A, two visits a week for four weeks; TRT B, one visit a week for four weeks; TRT C, two visits over four weeks; TRT D, one visit over four weeks; and TRT E, control, no therapy dog visits. Each visit lasted between $15 \pm \mathrm{SD}=5$ minutes in length and took place between 1130 and 1330. There was a one week washout period in-between each block. Therapy dog team visits were consistent on time and day of the week for each team, but due to volunteer basis, it varied between teams. Dog 1 and 2 visited on Mondays and Fridays, Dog 3 visited on Tuesdays and Thursdays, and Dog 4 visited on Mondays and Wednesdays. During this visit, the handler and dog met the nurses at a designated, quiet area. The session consisted of the nurses petting the therapy $\operatorname{dog}$ and conversing with the dog handler; visits were non-goal oriented. Throughout the duration of the study, the therapy dogs were not allowed to make any additional hospital visits, to control for the exact frequency of visits during the four week period.

Prior to the start of therapy dog visits, nurses were asked to fill out a general demographic survey, the Pet Attitude Scale-Modified survey, and the Lexington Attachment to Pet Scale survey. During pre- and postvisits, participants were asked to fill out the Maslach Burnout Inventory (MBI)-Human services survey, the Nursing Workplace Satisfaction Questionnaire, the Nursing Work Index-Revised questionnaire, and a visual analog scale.

\section{Statistical Analysis}

The surveys among nursing units were analyzed as a completely randomized design using the MIXED procedure of SAS version 9.4 (SAS Institute Inc., Cary, NC). Pre- and post-survey scores for each different treatment were analyzed using a $t$ test of SAS 9.4. Significance was set at $(P \leq 0.05)$, and the tendency was set at $(P \leq 0.10)$.

\section{RESULTS}

\section{Nursing Demographic}

The 24 nurses that participated in this study consisted of $16(66.66 \%)$ who were married, $2(8.33 \%)$ divorced, 4 (16.66\%) single, and $2(8.33 \%)$ who chose not to answer. Out of the participants, only one marked "yes" to smoking and $14(58.33 \%)$ noted that they consuming 1 to 3 alcoholic beverages a week. Two out of the 24 participating nurses did not own pets. When asked, 15 $(62.50 \%)$ of participants did not have any intentions of leaving their nursing unit, $1(4.16 \%)$ intended to leave their job within 12 months, and 8 (33.33\%) were unsure.

\section{Pet Attitude Scale-Modified and The Lexington Attached to Pet Scale}

Prior to the start of the treatments, the outpatient nurses were asked to complete the Pet Attitude ScaleModified and the Lexington Attachment Pet Scale to obtain a generalized picture of how the participants felt about pets. Nineteen (79.16\%) participants responded with, "strongly agree" when asked if they loved pets and strongly agreed that house pets add to their happiness in life. In addition, 21 out of 24 (87.50\%) strongly agreed that they would like to have a pet in their home; and strongly disagreed that having pets was a waste of money. In addition, $100 \%$ of the participants disagreed when asked if they hate animals. Lastly, 21 out of the $24(87.50 \%)$ participants, "strongly disagreed" that they were not very attached to their pets, with the remaining three participants, "somewhat disagreeing".

\section{Visual Analog Scale}

The visual analog scale is a 10-point scale, with zero meaning the highest possible level of feeling happy, energetic, relaxed, and calm, with a positive overall emotional well-being. A score of zero also indicates none at all for parameters including restlessness, fatigue, anxiety, depressed mood, and feeling a high level of stress. Thus, a reduction in post-treatment 
scores indicates a positive outcome. When comparing pre- and post-self-reported responses of the visual analog scale, TRT B had a significant decrease $\left(t_{80}=\right.$ -2.24; $P=0.02)$ on depression scores from pre- to posttreatment responses. In addition, TRT $B$ had a significant decrease $\left(t_{79}=-2.00 ; P=0.04\right)$ on overall emotional well-being scores. TRT A was significant $\left(t_{80}\right.$ $=-2.60 ; P=0.01)$ at reducing the post-self-reported scores of happiness.

When comparing numbers, more post-treatment responses were higher within TRT C and TRT D, with five out of 10; TRT E had six variables out of 10 (Table 2). In addition to the notable numeric comparison between treatments, there was a significant nursing unit effect on the responses (ANOVA: $\mathrm{F}_{3,94}=4.64 ; P<$ 0.05).

\section{Maslach Burnout Index (MBI)—Human Services Survey}

The survey question regarding personnel feeling drained at work was significantly different $\left(t_{15}=1.76 ; P\right.$ $=0.04)$ pre- and post-treatment for TRT A. The most common response to pre-therapy dog visits was "once a week"; however, after two weekly visits from a therapy dog over 4 weeks, the post-treatment response was "once a month or less."

The responses to the statement, "Working with people all day is really a strain for me," was significantly different between TRT A $\left(t_{17}=1.89 ; P=0.03\right)$ and TRT D $\left(t_{17}=-1.27 ; P=0.04\right)$ when comparing pre- and posttreatment responses. TRT A's most common pretreatment response was "once a week." After the 4 weeks of therapy dog visits, TRT A's most common response was "one a month or less." In addition, TRT D's most common pre-treatment response was "a few times a year or less," and after a single visit with a therapy dog over the course of 4 weeks, the responses were "once a month or less."

Lastly, the responses for TRT A's pre- versus posttreatment responses to the statements, "I feel frustrated by my job," and "I feel I'm working too hard on my job," were significantly different $\left(t_{17}=3.20 ; P=0.02\right.$ and $t_{17}=1.74 ; \quad P=0.03$, respectively). The post-treatment response by over half of the individuals was "a few times a year." In addition, TRT A's post-treatment responses for feeling frustrated at the job were "never," and "a few times a year." Both responses decreased from the severity of their responses before therapy dog visits.

\section{Nurse Working Index-Revised}

There was no significant difference among or within treatments for the Nurse Working Index-Revised questionnaire. However, there were a unit effects, with three out of the four staffing adequacy questions. "Enough time and opportunity to discuss patient care problems with other" was significantly different among units (ANOVA: $\mathrm{F}_{3,172}=3.50 ; \quad P=0.01$ ). "Enough registered nurses on staff to provide quality patient care" was highly significantly different among units (ANOVA: $\mathrm{F}_{7,175}=6.99 ; P<0.05$ ). Lastly, "Enough staff to get the work done" was highly significantly different among units (ANOVA: $\mathrm{F}_{7,178}=5.71 ; P<0.05$ ). There was also a significant different in regards to nurse-physician relation when asked about collaboration (ANOVA: $F_{7,177}$ $=2.03 ; P=0.05$ ). For all significantly different unit effects, nursing unit two had the highest, most agreeable responses, while nursing unit four had the lowest, most disagreeable responses. 


\begin{tabular}{|c|c|c|c|c|c|c|c|c|c|c|}
\hline & \multicolumn{2}{|c|}{ TRT A $^{1}$} & \multicolumn{2}{|c|}{ TRT B $^{2}$} & \multicolumn{2}{|c|}{ TRT C $^{3}$} & \multicolumn{2}{|c|}{ TRT D ${ }^{4}$} & \multicolumn{2}{|c|}{ TRT $E^{5}$} \\
\hline & Pre & Post & Pre & Post & Pre & Post & Pre & Post & Pre & Post \\
\hline Restlessness & 1.95 & $2.30^{b}$ & 2.40 & 2.55 & 2.52 & 2.33 & 1.88 & $2.27^{b}$ & 2.50 & $2.71^{b}$ \\
\hline Fatigue & 3.33 & 2.89 & 3.85 & 3.85 & 3.55 & 3.16 & 3.61 & $3.94^{b}$ & 3.72 & 3.57 \\
\hline Anxiety & 2.90 & $3.05^{b}$ & 3.80 & $3.90^{b}$ & 3.10 & 2.83 & 3.33 & $3.88^{b}$ & 3.11 & $3.21^{b}$ \\
\hline Depression & 1.76 & 2.00 & 2.70 & $1.95^{\mathrm{a}}$ & 2.05 & 2.05 & 2.00 & $2.72^{\mathrm{b}}$ & 2.22 & $2.64^{b}$ \\
\hline $\begin{array}{l}\text { High stress } \\
\text { level }\end{array}$ & 2.76 & 2.50 & 3.80 & 3.45 & 2.95 & 3.00 & 3.00 & $3.88^{b}$ & 3.11 & 3.07 \\
\hline Happy & 4.00 & $2.45^{\mathrm{a}}$ & 4.70 & 3.85 & 2.85 & $3.66^{b}$ & 3.57 & 3.05 & 4.00 & 3.85 \\
\hline Energetic & 3.90 & 3.20 & 4.40 & 4.15 & 3.60 & $3.88^{b}$ & 3.80 & 3.61 & 4.31 & 4.07 \\
\hline Relaxed & 4.04 & 3.30 & 4.70 & 4.50 & 3.52 & $3.66^{b}$ & 3.61 & 3.05 & 4.33 & $4.61^{b}$ \\
\hline Calm & 3.90 & 3.20 & 4.52 & 4.25 & 3.42 & $3.82^{b}$ & 3.52 & 3.27 & 3.89 & $4.21^{b}$ \\
\hline $\begin{array}{l}\text { Overall } \\
\text { emotional } \\
\text { well-being }\end{array}$ & 4.04 & 3.40 & 4.80 & $4.21^{\mathrm{a}}$ & 3.57 & $3.77^{b}$ & 3.80 & 3.05 & 4.15 & $4.28^{b}$ \\
\hline
\end{tabular}

Table 2. Pre- and Post-Self-Reported Visual Analog Scores for Each Treatment (the smaller the number means the best possible/none at all). TRT, treatment; ${ }^{1}$ Treatment A: therapy dog visit twice a week for 4 weeks; ${ }^{2}$ Treatment B: therapy dog visit once a week for 4 weeks; ${ }^{3}$ Treatment $C$ : therapy dog visit twice over 4 weeks; ${ }^{4}$ Treatment $D$ : therapy dog visit once over 4 weeks; ${ }^{5}$ Treatment E: control (no therapy dog visit).

a Significantly decreased from pre- to post-treatment scores $(P<0.05) .{ }^{b}$ Numerically increased post-self-reported scores compared to pretreatment scores (zero meaning none at all or best possible).

\section{DISCUSSION}

The responses in the Pet Attitude Scale- Modified and Lexington Attachment to Pet Scale confirmed that over $80 \%$ of the participants in the study believe that owning pets contribute to their happiness and having a pet in the house adds to their overall happiness in life. These results suggest that those who participated in the study had a pet that they were attached to and thought it added happiness to their life. Thus, the authors conclude that the addition of a dog as stress relief event would not trigger a stress response.

The visual analog scale analysis supports the theory that therapy dog visits can help reduce compassion fatigue. In TRT A, in which a therapy dog visited twice a week for 4 weeks, a significant increase in the feeling of happiness was indicated. In addition, TRT B, which utilized a therapy dog visit once a week for 4 weeks, was able to markedly reduce self-reported responses of depression and improved overall emotional well-being. Consequently, in TRT E, the control/no therapy dog visit group, the least amount of improvement in the nursing units' visual analog scale was seen, with six parameters being self-reported as worse. In addition, TRT C, in which therapy dogs visited twice total over 4 weeks, and TRT D, in which therapy dogs visited just once over 4 weeks, numerically had an increase in five 
visual analog scale scores, indicating that these five parameters worsened from pre- to post-treatment responses.

TRT A was able to make a substantial positive effect on the post-treatment responses of the MBI-Human Service Survey, which supports the hypothesis that therapy dog visits can help combat nurses feeling drained after work, strained during work, and overall less frustrated about their job. These preliminary results support the theory that having staff stress-relief events in the form of therapy dog visits, roughly once a week, can help nurses cope with compassion fatigue and burnout. The Nurse Working Index-Revised questionnaire; however, did not show any substantial effects from treatments. In contrast, there was a unit effect for staff adequacy questions and nurse-doctor collaboration, with unit two having the most agreeable responses and unit four having the most disagreeable responses. This suggests that the same level of collaboration and staffing are significantly different and the unit the nurse works in could greatly affect their risk for compassion fatigue, burnout, and overall wellbeing. Therefore, while therapy dog visits may be effective at reducing compassion fatigue and burnout, they may not be helpful in the satisfaction level of nurses and their relationships with physicians and other nurses.

Past literature supports our findings. Studies have been conducted observing the beneficial relationship that animals have with humans (Nimer and Lundahl, 2007). It has been noted that visits from dogs can considerably reduce anxiety levels and improve individual coping ability, which are both useful tools in overcoming compassion fatigue and burnout (Baker and Dawson 1998; Berget et al., 2008).

One major limitation to this study is that the nursing population was strictly outpatient nurses who were not always available, due to schedule or vacation time, to attend the therapy dog visits. While researchers prescreened nurses who stayed in the same speciality area, worked full time, and we were willing to attend, finding a time and date that worked for the dog and handler and all the participating nurses in each unit posed a challenge. A second limitation was the nursing unit effect. The participants within each unit voluntarily joined the study. However, naturally, some units are always a high stress area and nurses may have had individual hardships within the unit during the study period. In addition to this, there is potential bias from using survey responses. While the questionnaires used in this study are validated, the authors recognize that the nurses' perception can add bias and to strengthen the study, objective parameters such as, oxytocin, cortisol, and heart rate variability, could be included in future studies.

\section{CONCLUSION}

This small, novel study sheds light on potential avenues to assist nurses in overcoming compassion fatigue and burnout; however, larger studies should be conducted for a longer duration of time and be distributed more evenly among nursing units. In addition, it would add another point of view to introduce this staff stress event to inpatient nurse populations. This study supports the hypothesis that visits, most effectively weekly visits, from a therapy $\mathrm{dog}$, can help alleviate stress and feeling frustrated, drained, and overwhelmed from working in the nursing field. It would be interesting to take this study further to observe the mechanism behind this conclusion.

\section{ACKNOWLEDGEMENTS}

This publication was made possible by the Mayo Clinic CTSA through grant number UL1TR002377 from the National Center for Advancing Translational Sciences (NCATS), a component of the National Institutes of Health (NIH).

\section{REFERENCES}

Aiken, L. H., Clarke, S. P., Douglas, M., and Sloane, D. M. 2002. Hospital nurse staffing

and patient mortality, nurse burnout, and job dissatisfaction. Journal of American Medicine Association 288(16): 1987-1993. doi:10.1001/jama.288.16.1987.

Aiken, L. H., Cimiotti, J. P., Sloane, D. M., Smith, H. L., Flynn, L., and Neff, D.F. 2011. 
The effects of nurse staffing and nurse education on patient deaths in hospitals with different nurse work environments. Medical Care 49(12): 1047-1053. Doi. 10.1097/MLR.0b013e3182330b6e.

Barker, S. B., and Dawson, K. S. 1998. The effects of animal-assisted therapy on anxiety ratings of hospitalized psychiatric patients. Psychology Series 49(6): 797-802.

Beetz, A., Julius, H., Turner, D., and Kotrschal, K. 2012. Effects of social support by a dog on stress modulation in male children with insecure attachment. Frontier Psychology 3: 352.

Berget, B., Ekeberg, O., and Braastad, B. O. 2008. Animal-assisted therapy with farm animals for person with psychiatric disorders: Effects on self-efficacy, coping ability, and quality of life, a randomized controlled trial. Clinical Practice Epidemiology Mental Health 4(9).

Nimer, J., and Lundahl, B. 2007.Animal-assisted therapy: A meta-analysis. Anthrozoology 20(3): 225-238.

Shimizutani, M., Odagiri, Y., Ohya, Y., Shimomitsu, T., Kristensen, T. S., Maruta, T. M. and Iimori, M. 2008. Relationship of nurse burnout with personality characteristics and coping behaviors. Industry Health 46: 326-335.

Vahey, D. C., Aiken, L. H., Sloane, D. M., Clarke, S. P., Vargas, D. 2004. Nurse burnout andpatient satisfaction. Medical Care 42(2 Suppl): II57-II6.

\section{Cc) creative}

This paper has been published by

Pet Behaviour Science

under a Creative Commons license

4.0 Non-comercial - Share Alike - Attribution

As an open access journal, it is free of charges for both authors and readers

wWw.petbehaviourscience.org 